VARGAS TO; ALVES EP; ABBOUD ACS; LEAL MAA; CARMO MGF. 2015. Diversidade genética em acessos de tomateiro heirloom. Horticultura Brasileira 33: 174-180. DOI - http://dx.doi.org/10.1590/S0102-053620150000200007

\title{
Diversidade genética em acessos de tomateiro heirloom
}

Thiago O Vargas ${ }^{1}$; Elisabete P Alves²; Antonio CS Abboud ${ }^{2}$; Marco AA Leal ${ }^{3}$; Margarida GF Carmo²

${ }^{1}$ UTFPR, Depto. Ciências Agrárias, Via do Conhecimento km 01, 85503-390 Pato Branco-PR; thiagovargas@utfpr.edu.br; ${ }^{2} U F R R J$,

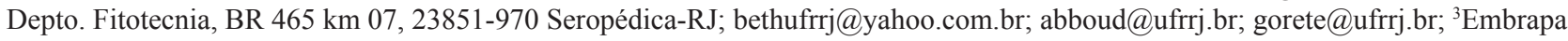
Agrobiologia, BR 465 km 07, 23851-970 Seropédica-RJ; marco.leal@embrapa.br

\section{RESUMO}

O objetivo deste trabalho foi avaliar a diversidade genética de acessos de tomates heirloom da coleção do Departamento de Fitotecnia da UFRRJ. A similaridade entre acessos foi determinada por meio de análise de componentes principais seguida de análise de agrupamento, utilizando como variáveis os descritores do IPGRI (International Plant Genetic Resources Institute). No período de maio a setembro de 2004 foram cultivados 22 acessos de tomate, sendo 10 acessos de tomateiro de frutos tipo cereja e 12 de frutos grandes, onde em cada grupo havia acessos heirloom assim como cultivares locais. Os dados oriundos de caracteres quantitativos foram submetidos à análise de componentes principais e seguidos de análise de agrupamento, pelo método 'Ward's minimum variance'. Os dados oriundos de caracteres qualitativos foram transformados em matriz binária, a partir da qual foram calculados os índices de similaridade de Jaccard e submetidos à análise de agrupamento usando o método UPGMA (método da média aritmética não ponderada), que deu origem aos dendogramas de similaridade. Mediante análise dos resultados foi observada a formação de agrupamentos onde os acessos locais se separavam dos genótipos heirloom, indicando variabilidade genética. As análises com os dois tipos de descritores, quantitativos e qualitativos, agruparam os acessos de forma que aqueles de origem local possuíam baixas similaridades com os heirloom importados. Embora os agrupamentos formados pelos dois métodos não sejam idênticos, os dois tipos de análises, em conjunto, são adequados para caracterizações exploratórias em coleções de tomateiro, devido a sua rapidez e praticidade.

\begin{abstract}
\section{Genetic diversity in heirloom tomato genotypes}

The objective of this research was to study the genetic diversity of a group of heirloom tomatoes genotypes from Universidade Federal Rural do Rio de Janeiro, Brazil, using descriptors recommended by the Bioversity International Institute (formerly International Plant Genetic Resources Institute). Similarity between genotypes was quantified by principal component analysis followed by clustering methods. Ten genotypes of large-fruit tomatoes and twelve of cherrytype tomatoes were grown from May to September 2004. In each fruit size group, both heirlooms and commercial genotypes were present. Quantitative data were submitted to principal component analyses followed by cluster analyses by the Ward's minimum variance method. Qualitative data were transformed into a binary matrix, from which similarity indexes (Jaccard) were generated and submitted to a cluster analysis by the UPGMA method in order to generate dendrograms. Results showed the formation of clusters where local and commercial genotypes were separated from imported heirlooms, indicating diversity. Clusters formed by either qualitative or quantitative variables discriminated these two groups, although the clusters formed were not identical. Hence, these two techniques combined are adequate to exploratory analyses of large numbers of genotypes, since they are fast and easy to perform.
\end{abstract}

Keywords: Solanum spp., germplasm, multivariate analyses.

(Recebido para publicação em 27 de maio de 2014; aceito em 17 de dezembro de 2014) (Received on May 27, 2014; accepted on December 17, 2014)

\begin{abstract}
$\mathrm{A}^{\mathrm{c}}$ exploração da agrobiodiversidade pode trazer diversos benefícios na concepção de novos sistemas de produção. No caso de tomateiros, há um grande potencial para expandir o número de cultivares disponíveis e assim possibilitar maiores opções para produtores e consumidores.

$\mathrm{O}$ fruto do tomateiro possui enorme variabilidade de forma, cor, textura e várias outras características sensoriais
\end{abstract}

como o aroma e sabor adocicados, frutados (frescos e intensos) ou ácidos (Rocha et al., 2013a, 2013b). No entanto, o desconhecimento e/ou a preferência dos consumidores torna o mercado restrito, com oferta de poucos grupos deste produto. $\mathrm{O}$ mercado de sementes disponibiliza uma variabilidade restrita de cultivares de tomate, geralmente híbridos, de alto custo, com frutos grandes de coloração vermelha, restringindo as escolhas dos produtores e consumidores.

Recentemente, Rocha et al. (2013b) verificaram que dentre 16 atributos sensoriais propostos, o sabor característico do tomate, a resistência da casca à mastigação, o aroma doce, a forma arredondada, a suculência, o aroma e sabor ácidos, a firmeza do fruto, a crocância, a presença de ombro verde e o sabor doce, foram aqueles que mais contribuíram para a variância em um es- 
tudo com dez acessos de tomate cereja, e foram os que mais contribuíram para agrupar os acessos.

Na Europa e nos Estados Unidos, as hortaliças heirloom (termo da língua inglesa que se refere aos acessos de plantas cultivadas, principalmente hortaliças, passadas de geração a geração, por famílias de agricultores) têm se popularizado nos meios de produtos orgânicos e outros nichos, preocupados com qualidade alimentar e sustentabilidade da produção. As hortaliças heirloom podem ser facilmente encontradas em todos os grandes centros dos EUA e muitos países da União Européia (Gonçalves et al., 2009). Da mesma forma, não é incomum encontrar nos mercados de sementes uma extensa oferta dessas. A cultura heirloom é normalmente associada à agricultura familiar, aos métodos orgânicos de produção, à distribuição local e ao baixo custo de sementes. No Brasil, esse material genético é ainda pouco conhecido e explorado. Definições e citações sobre plantas ou cultivares heirloom são raras na literatura agronômica brasileira. O termo em português que mais se aproxima desta palavra seria "semente crioula". Há poucas publicações sobre o assunto em língua inglesa, onde se verifica que as variedades heirloom têm crescido em importância, devido a iniciativas de consumidores e de pequenos produtores, mais do que de pesquisadores (Nazarea, 2005; Jordan, 2007).

Ao longo dos anos, houve o aprimoramento das técnicas estatísticas utilizadas para análise de dados referentes à caracterização e à avaliação de germoplasma e, no Brasil, os descritores quantitativos estão entre os mais estudados em hortaliças. O aumento no uso de técnicas multivariadas para quantificação da divergência genética tem sido verificado, pois, essas análises permitem considerar simultaneamente inúmeras características (Sudré et al., 2007), mas também as relacionadas a produtividade e desempenho agronômico. Trabalhos com outras hortaliças evidenciam que características relacionadas à produção, tais como o número de vagens por parcela em plantas de feijão de vagem (Abreu et al., 2004) e número de frutos por planta em Capsicum spp. (Bento et al.,
2007), não contribuem na discriminação de acessos geneticamente distintos. Por isso, outras características como descritores morfológicos diversos devem ser usados, apesar desses caracteres de produção serem úteis na identificação de genótipos.

A utilização de métodos multivariados de agrupamento de germoplasma, com base em caracteres morfológicos, constitui uma ferramenta eficaz no estudo exploratório da variabilidade genética. Estudos nesse sentido foram realizados com acessos de tomate por Karasawa et al. (2005) e com outras hortaliças, como o quiabeiro (Omonhinmin \& Osawaru, 2005), alho (Trani et al., 2005), caupi (Oliveira et al., 2003), feijão de vagem (Maluf et al., 1983; Abreu et al., 2004), taro (Pereira et al., 2003), pimenta e pimentão (Sudré et al., 2005). Nesses, ambos os caracteres qualitativos e quantitativos podem ser usados para se explorar a divergência genética ou similaridade entre acessos.

A técnica de componentes principais é empregada na seleção de descritores de várias espécies como mandioca (Pereira et al., 1992), caju (Barros, 1991), cacau (Dias et al., 1997), cupuaçu (Alves et al., 2003), guandu (Santos et al., 1995), capim-elefante (Daher et al., 1997), açaizeiro (Oliveira et al., 2006) e espécies do gênero Paspalum (Strapasson et al., 2000).

Os objetivos deste trabalho foram avaliar a diversidade genética e quantificar a similaridade em acessos de tomateiro, com base em descritores qualitativos e quantitativos, promissores para a produção orgânica.

\section{MATERIAL E MÉTODOS}

Em maio de 2004 foram semeados 22 acessos de tomateiro da coleção do Departamento de Fitotecnia da UFRRJ, sendo 10 acessos de frutos tipo cereja e 12 de frutos grandes, com o transplantio em junho. O experimento foi conduzido na Estação Experimental da PESAGRO RIO, em Seropédica-RJ, situada na Baixada Fluminense (2245'S, 4340'O, $26 \mathrm{~m}$ de altitude). A região apresenta clima tipo Aw, segundo a classificação de Köppen, com verão úmido e inverno seco. A temperatura média anual é de $24,6^{\circ} \mathrm{C}$ e a precipitação média anual de $1200 \mathrm{~mm}$, sendo os meses de julho e agosto os mais secos, segundo dados coletados na estação meteorológica local, situada na Estação Experimental da PESAGRO RIO, em Seropédica-RJ.

As mudas foram produzidas em bandejas de polipropileno, com 128 células, preenchidas com substrato preparado a partir de terra argilosa adicionada de esterco de curral $(3: 1 \mathrm{v} / \mathrm{v})$ e mantidas em casa de vegetação. Os acessos do tipo cereja foram transplantados para o campo e os de frutos grandes em casa de vegetação. $\mathrm{O}$ cultivo em ambiente protegido foi necessário pelo fato dos tomates de frutos grandes serem muito suscetíveis a brocas e ao apodrecimento causado por chuvas, o que não ocorre com os do tipo cereja.

Em ambos locais, as plantas foram dispostas em parcelas com seis indivíduos e quatro repetições, distribuídas em blocos ao acaso. As áreas foram preparadas com trator e coveadas no espaçamento de 1,0x0,4 m entre plantas. As plantas receberam adubação por cova com $1 \mathrm{~L}$ de esterco de curral, $30 \mathrm{~g}$ de termofosfato e $30 \mathrm{~g}$ de cinza, seguindo as recomendações para a agricultura orgânica (Leal et al., 2007). No decorrer do ciclo, foi realizada uma adubação de cobertura com esterco de frango (100 g/cova). A cultura foi conduzida mantendo-se as plantas amarradas ao longo de duas guias com fitilhos de plástico, realizando desbrotas conforme a necessidade. As colheitas foram semanais, de setembro a outubro, perfazendo um total de sete colheitas na casa de vegetação e cinco no campo, devido ao aparecimento do fungo Phytophthora infestans. A ocorrência dessa doença diminuiu o número de colheitas, que pode chegar a dez no caso do tomate cereja.

Foi realizada a caracterização descritiva nos dois grupos de acessos de tomateiro, sendo os de frutos do tipo grande: Santa Clara e Kada Gigante (cultivares comerciais nacionais); Seculus (híbrido nacional); Bush Roma (obtida a partir da cultivar heirloom Roma de origem italiana), e Mule Team, Golden Queen, Cherokee A, Eva Purple Ball, Jefferson Giant, Mortgage Lifter, Big Beef, Amish Paste (Heirlooms oriundos dos EUA). 
No outro grupo, os acessos do tipo cereja: 'Viçosa' (obtido de produtor no município de Viçosa); 'Perinha local' (obtido de produtor no município de Seropédica); Black Prince, Besser, Bonny Best, Tigerella (Heirlooms oriundos da firma 'Kokopelli', na França); Rosa, E, $\mathrm{K}$, A (heirlooms oriundos dos EUA).

De acordo com o International Plant Genetic Resources Institute (IPGRI, 1996), utilizaram-se os seguintes descritores quantitativos: comprimento do internódio ( $\mathrm{mm}$ ), comprimento do fruto ( $\mathrm{mm})$, número de lóculos, espessura do pericarpo $(\mathrm{mm})$, largura do fruto $(\mathrm{mm})$, comprimento do pedicelo (mm), seção cilíndrica do fruto-tamanho (mm), seção cilíndrica do fruto-largura (mm). À exceção do número de lóculos, as demais variáveis foram obtidas com o auxílio de paquímetro.

Os descritores qualitativos usados no presente estudo foram pêlo de pubescência do estilete (ausência ou presença), tamanho do fruto (muito pequeno a muito grande), forma predominante do fruto (achatado a cilíndrico), cor do fruto imaturo (escuro: com ombro verde presente a claro: com ombro verde ausente), cor da pele do fruto maduro (transparente a amarela/tangerina/vermelha), intensidade da cor da polpa (clara, média, escura), seção transversal do fruto (redonda, angular, irregular), presença de pedicelo com joelho (ausência ou presença), presença de cicatriz do cálice (ausente, leve, média, acentuada), tamanho da cicatriz (pequena, média, grande), rachadura radial no fruto (ausente a severa), rachadura concêntrica no fruto (ausente a severa), presença de faces no fruto (sutil a severa), tipo de caule (inflexível, flexível, ambos), pubescência do caule (ausente a forte), número de folhas abaixo da primeira inflorescência (poucas, média, muitas), atitude da folha (semi-ereta, horizontal, caída), tipo de folha (conforme figuras 1, 2, 3, 4), coloração da antocianina nas nervuras das folhas (ausente ou presente), tipo de inflorescência (geralmente unípara, geralmente multípara), tamanho da planta no florescimento (pequena, média, grande), cobertura foliar (fraca, regular, boa), variabilidade de tamanho (1: uniforme a 9: extremamente variável), formato secundário do fruto na colheita (achatado a cilíndrico), intensidade do verde escuro antes da maturação (leve, médio, forte), comprimento do pedicelo (curto, comprido), área do pedicelo (achatado a forte depressão), formato da região apical (ponto, estrelado, linear, irregular), condição da região apical do fruto: cicatriz estilar (aberta, fechada), presença de manchas, pintas coloridas no fruto durante o desenvolvimento (1: sem manchas a 9: muitas manchas), podridão apical no fruto, formato da cicatriz estilar (fruto irregular, achatada, pontuda), cor exterior do fruto (Munsell color charts for plant tissues), observados de acordo com a proposta por IPGRI (1996). Nas avaliações dos frutos priorizou-se a utilização de todos descritores, e assim retirando-se a média referente a cada acesso.

Todos os dados quantitativos foram submetidos à análise de componentes principais e análise de fatores (utilizando-se PROC FACTOR) e de agrupamento utilizando-se o método Ward's minimum variance (PROC CLUSTER) do pacote estatístico SAS (1999). Os dados de todos os descritores qualitativos foram transformados em matriz binária, a partir da qual foram calculados os índices de similaridade de Jaccard e submetidos à análise de agrupamento, pelo método UPGMA (método da média aritmética não ponderada), pelo pacote NTSYS, originando os dendogramas de similaridade dos acessos.

\section{RESULTADOS E DISCUSSÃO}

A partir da avaliação de variáveis descritivas em tomates de fruto tipo grande e tipo cereja, verifica-se a presença de variabilidade entre os acessos avaliados (Tabela 1). As variáveis 'cor do fruto' e 'cor da polpa' merecem atenção especial, visto que são as mais importantes para a escolha dos consumidores e que mais influenciaram nas análises sensoriais de tomates de fruto cereja, realizadas pelos autores junto a consumidores de diferentes perfis (dados não apresentados). Os acessos A e Black Prince, de seção angular e coloração escura, destacaram-se dos demais, por serem fora do padrão normalmente aceito no mercado brasileiro. $\mathrm{O}$ acesso Rosa se mostrou bastante promissor ao produtor por ter polpa firme e para o consumidor pela coloração branca quando imaturo e rosa quando maduro.

Alguns acessos de frutos grandes se destacaram por não terem frutos de polpa vermelha, cor mais comumente encontrada e aceita no Brasil. É o caso do Golden Queen com polpa fortemente amarela, do Mule Team, rosa-alaranjada e do Cherokee A, rosada intensa. Estas cores podem ser um atrativo para o consumidor que deseja proporcionar efeitos ornamentais em pratos de saladas. Quanto à forma, Cherokee A, Mule Team e Jefferson Giant são bastante irregulares e com muitos lóculos, o que resulta quando fatiado em seções bastante disformes, podendo ser uma limitação ao mercado. A forma de coração bastante diferenciada do acesso Amish Paste, seu tamanho grande e espessura da polpa bastante larga, tornam esse acesso, de coloração vermelho intensa, bastante atrativo a um consumidor que deseja produzir molhos.

A similaridade encontrada para os acessos de frutos grandes foi de 50\% (Figura 1), enquanto que para os frutos do tipo cereja, o maior valor foi de $67 \%$ (Figura 2). Esses são valores baixos de similaridade, o que demonstra a diversidade genética do grupo de acessos estudados.

Para os acessos de frutos grandes, houve a formação de seis agrupamentos, considerando o limite de $34 \%$ de similaridade. $\mathrm{O}$ primeiro agrupamento foi composto por Santa Clara, Seculus, Kada Gigante e Bush Roma, onde todos são acessos melhorados; o segundo, pelos acessos Cherokee A, Eva Purple Ball, Mortgage Lifter e Big Beef, onde todos são acessos heirloom e de frutos vermelhos. Os demais quatro acessos, Jefferson Giant, Mule Team, Golden Queen, e Amish Paste também heirlooms, cada um constituiu-se num agrupamento separado (Figura 1).

$\mathrm{O}$ acesso Amish Paste foi o mais contrastante de todos os demais. Trata-se de um material com frutos grandes e com forma de coração, altamente promissor por apresentar boa produtividade e estabilidade na produção, com frutos de polpa espessa e poucas sementes, de excelente aceitação no mercado.

Nos acessos de frutos tipo cereja, 
houve a formação de cinco agrupamentos, considerando o limite de $40 \%$ de similaridade. O primeiro foi formado pelos acessos 'Viçosa', Besser, E, 'Perinha local' e K e A. Nesse agrupamento, se enquadraram os dois acessos nacionais ('Viçosa' e 'Perinha' local) e os demais quatro agrupamentos foram formados cada um por um dos acessos, Black Prince, Bonny Best, Rosa e Tigerella (Figura 2). As características usadas nesta descrição são, na sua maioria, relativas à morfologia das plantas, enquanto que as características dos frutos estão melhor descritas pelas variáveis quantitativas, usadas na análise de componentes principais (Figuras 3 e 4).

Em relação aos acessos de frutos grandes, a análise de componentes principais revelou que os descritores comprimento do pedicelo e seção cilíndrica do fruto foram aquelas responsáveis pela maior variância (27\%) e correlacionadas ao componente principal 1 (Figura 3). O componente principal 2, responsável pela segunda maior variância $(25 \%)$, se correlacionou aos descritores comprimento do internódio e espessura do pericarpo. De acordo com esses dois componentes, houve a formação de três agrupamentos. O primeiro formado pelos acessos Big Beef, Mule Team e Seculus, por apresentarem maiores comprimento do pedicelo e seção cilíndrica do fruto. O segundo agrupamento foi formado pelos acessos Jefferson Giant, Amish Paste e Kada Gigante, por apresentarem maiores comprimento do internódio e maior espessura do pericarpo. O terceiro agrupamento foi composto pelos demais acessos, Santa Clara, Golden Queen, Mortgage Lifter e Eva Purple Ball, os quais apresentaram valores baixos para as variáveis associadas aos dois componentes principais. Embora totalmente independente da primeira análise, verifica-se que acessos como Amish Paste e Jefferson Giant, estão próximos entre si e bem distantes de Santa Clara nos dois tipos de análise. Já o acesso Bush Roma, destaca-se dos demais, devido suas características morfológicas diferentes dos demais acessos, inerentes às cultivares industriais.

Para os acessos de fruto tipo cereja (Figura 4), a análise de componentes principais revelou que o descritor

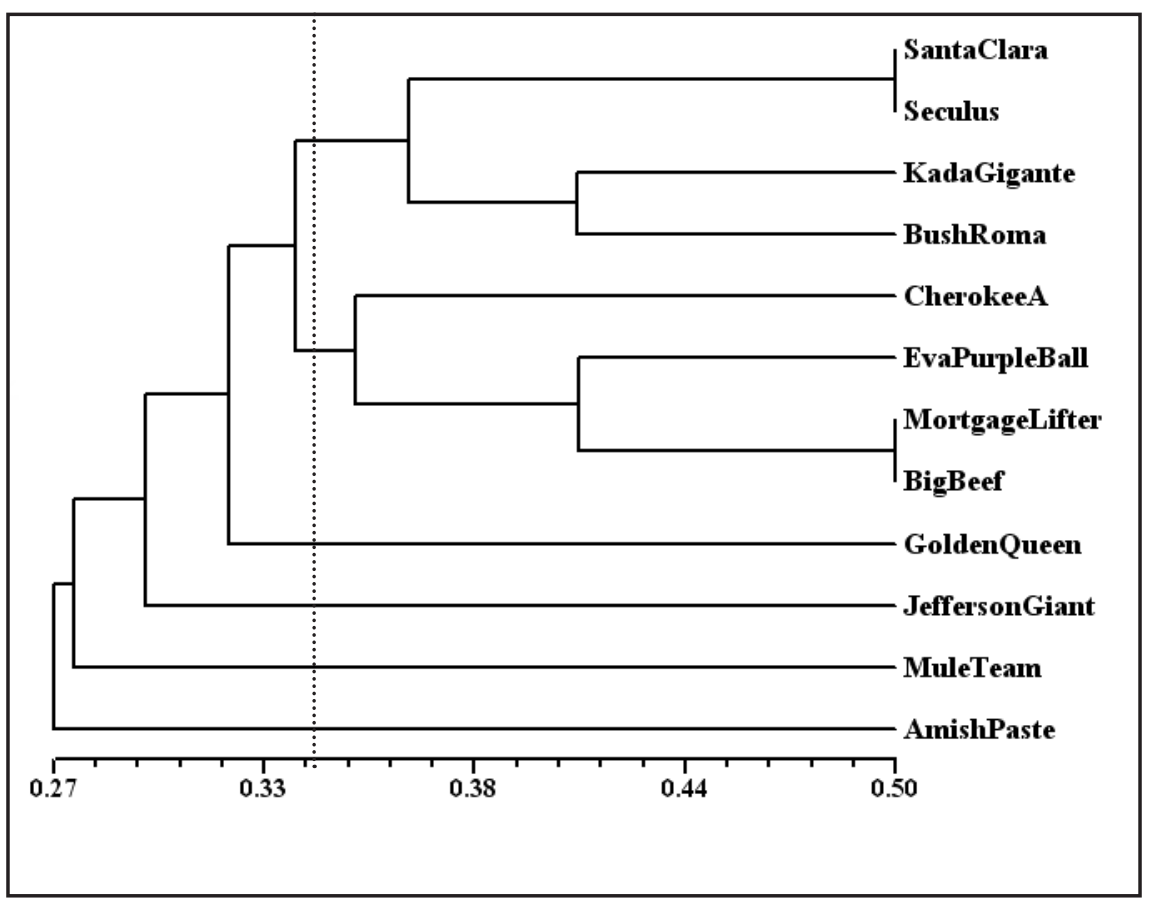

Figura 1. Dendograma de similaridade entre acessos de tomateiro de frutos grandes, formado a partir de variáveis qualitativas (dendogram of similarity among large-fruit acccessions of tomato based on qualitative variables). Seropédica, UFRRJ, 2004.

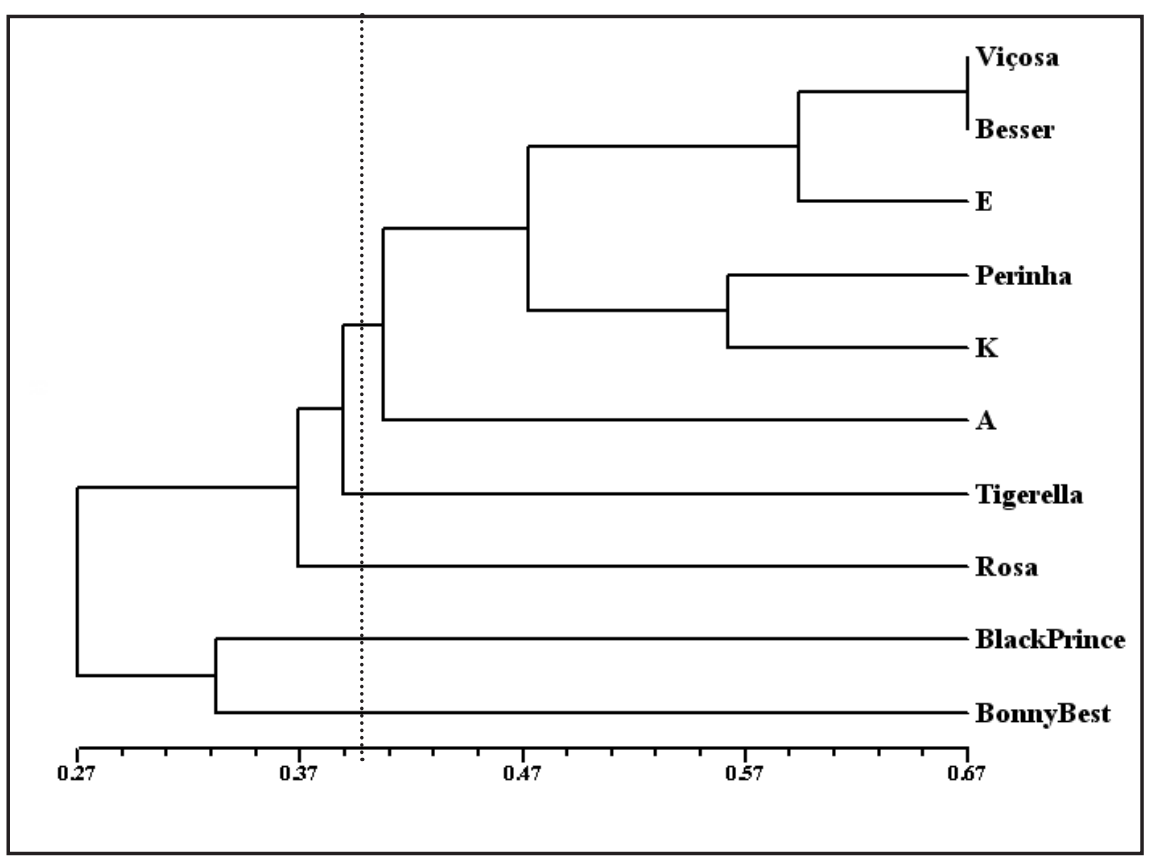

Figura 2. Dendograma de similaridade entre acessos de tomateiro de frutos do tipo cereja, formado a partir de variáveis qualitativas (dendogram of similarity among accessions of tomato with cherry-type fruits based on qualitative variables). Seropédica, UFRRJ, 2004.

firmeza do fruto maduro esteve correlacionado ao componente principal 1 (44\% da variância), e que os descritores tamanho do fruto e comprimento de pedicelo correlacionaram-se ao componente principal 2 (33\% da variância). Um agrupamento foi composto pelos acessos Black Prince, Bonny Best e A (grupo II) que apresentaram valores altos para comprimento do internódio, seção cilíndrica dos frutos, comprimento do pedicelo e tamanho do fruto. Este agrupamento esteve em posição contrastante ao agrupamento formado 


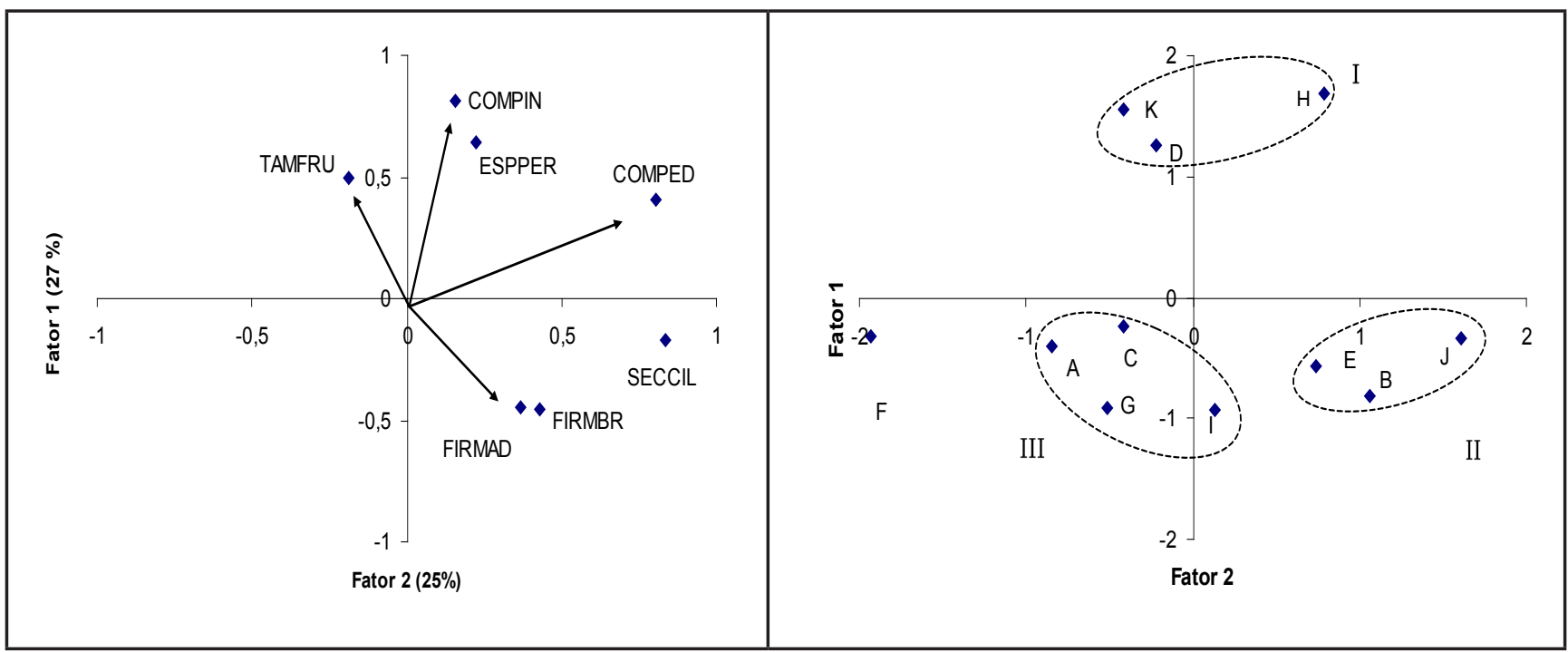

Figura 3. Resultado da análise de componentes principais e de agrupamento, a partir de variáveis descritivas quantitativas para acessos de frutos grandes (results of principal component analysis and clustering from quantitative descriptive variables for large-fruit tomato accessions). FIRMAD= firmeza do fruto maduro (firmness of ripe fruit); FIRMBR= firmeza breaker (breaker firmness); COMPIN= comprimento do internódio (internode lenght); ESPPER= espessura do pericarpo (pericarp thickness); TAMFRU= tamanho do fruto (fruit size); COMPED= comprimento do pedicelo (pedicel lenght); $\mathrm{SECCIL}=$ seção cilíndrica (cilindrical section); $\mathrm{A}=\mathrm{Santa} \mathrm{Clara}$; $\mathrm{B}=\mathrm{Mule}$ Team; $\mathrm{C}=\mathrm{Golden}$ Queen; D= Kada Gigante; E= Seculus; F= Bush Roma; G= Eva Purple Ball; H= Jefferson Giant; I= Mortgage Lifter; J= Big Beef; K= Amish Paste. Seropédica, UFRRJ, 2004.

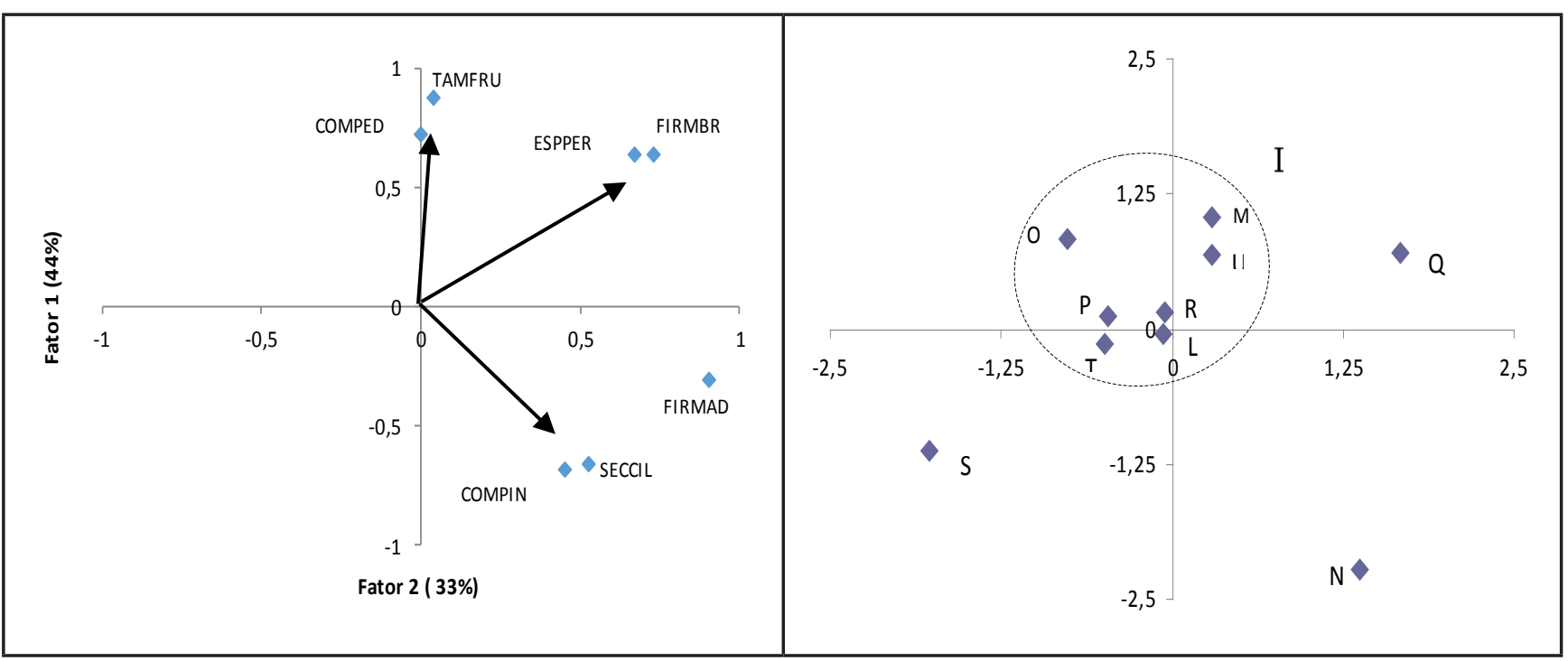

Figura 4. Resultado da análise de componentes principais e de agrupamento, a partir de variáveis descritivas quantitativas para acessos de frutos do tipo cereja (results of principal component analysis and clustering from quantitative descriptive variables for tomato of cherry-type fruits); FIRMAD= firmeza do fruto maduro (ripe fruit firmness); FIRMBR= firmeza breaker (breaker firmness); COMPIN= comprimento do internódio (internode lenght); ESPPER= espessura do pericarpo (pericarp thickness); TAMFRU= tamanho do fruto (fruit size); COMPED= comprimento do pedicelo (pedicel lenght); $\mathrm{SECCIL}=$ seção cilíndrica (cilindrical section); $\mathrm{L}=\mathrm{Viçosa} ; \mathrm{M}=\mathrm{Black} \mathrm{Prince}$; $\mathrm{N}=\mathrm{Besser}$; $\mathrm{O}=$ Bonny Best; $\mathrm{P}=$ Perinha; $\mathrm{Q}=$ Rosa; $\mathrm{R}=$ Tigerella; $\mathrm{S}=\mathrm{E} ; \mathrm{T}=\mathrm{K} ; \mathrm{U}=\mathrm{A}$. Seropédica, UFRRJ, 2004.

pelo acesso Besser (grupo III), que teve baixos valores para essas variáveis. $\mathrm{O}$ acesso Rosa formou um agrupamento (grupo I), apresentando altos valores para as variáveis associadas ao componente 2, ou seja, maior firmeza do fruto maduro, contrastando com um outro agrupamento formado pelo acesso ' $E$ ' (grupo IV). O quinto agrupamento, formado pelos demais acessos, apresentou valores intermediários para as variáveis associadas aos dois componentes. Assim como ocorreu com os acessos de frutos grandes, não houve concordância na maioria dos agrupamentos gerados pelas análises de variáveis qualitativas e quantitativas. Por exemplo, os acessos Rosa, Besser e Black Prince, ocupam agrupamentos diferentes nas duas análises.

A maioria das espécies exploradas agronomicamente teve sua diversidade 
Tabela 1. Resultado de alguns descritores em tomates de fruto tipo grande e tipo cereja (results of some descriptors on large-fruit and cherry-type tomatoes). Seropédica, UFRRJ, 2004.

\begin{tabular}{|c|c|c|c|c|c|}
\hline Genótipos & Forma do fruto & $\begin{array}{c}\text { Seção transversal } \\
\text { do fruto }\end{array}$ & $\begin{array}{l}\text { Número de } \\
\text { lóculos }\end{array}$ & $\begin{array}{l}\text { Cor exterior } \\
\text { do fruto }\end{array}$ & Cor da polpa \\
\hline \multicolumn{6}{|c|}{ Frutos tipo grande } \\
\hline Santa Clara & comprido redondo & redonda & $2-3$ & 10R $5 / 10$ & vermelho \\
\hline Mule Team & achatado & irregular & $8-9$ & 10R $5 / 10$ & $\begin{array}{l}\text { rosa e } \\
\text { laranja }\end{array}$ \\
\hline Golden Queen & levemente achatado & angular & 4 & $2,5 Y 8 / 10$ & amarela \\
\hline Kada Gigante & cilíndrico & angular & $2-3$ & $10 \mathrm{R} 5 / 10$ & tangerina e vermelha \\
\hline Seculus & levemente achatado & redonda & $3-5$ & 10R $5 / 10$ & tangerina e vermelha \\
\hline Bush Roma & cilíndrico & angular & 3 & 10R $5 / 10$ & vermelha \\
\hline Cherokee A & achatado & irregular & $5-8$ & $10 \mathrm{R} 4 / 10$ & rosa e vermelha \\
\hline Eva Purple Ball & redondo & redonda & $5-6$ & 5R 5/8 & vermelha \\
\hline Jefferson Giant & coração & irregular & $6-9$ & 5R 5/8 & tangerina e vermelha \\
\hline Mortgage Lifter & levemente achatado & redonda & $6-11$ & 2,5R 4/10 & vermelha \\
\hline Big Beef & levemente achatado & redonda & $6-7$ & 10R $5 / 10$ & vermelha \\
\hline Amish Paste & coração & angular & $4-8$ & 10R $4 / 10$ & vermelha \\
\hline \multicolumn{6}{|c|}{ Frutos tipo cereja } \\
\hline Viçosa & redondo & redonda & 2 & $10 \mathrm{R} 4 / 10$ & tangerina e vermelha \\
\hline Black Prince & comprido redondo & angular & $4-5$ & $2,5 Y R \quad 4 / 6$ & tangerina/marron \\
\hline Besser & redondo & redonda & 2 & $10 \mathrm{R} 4 / 10$ & tangerina e vermelha \\
\hline Bonny Best & comprido redondo & redonda & $6-7$ & $10 \mathrm{R} 5 / 10$ & tangerina e vermelha \\
\hline Perinha & pêra & redonda & 2 & 10R $5 / 10$ & tangerina e vermelha \\
\hline Rosa & ameixa & angular & 2 & $5 \mathrm{R} 5 / 10$ & rosa \\
\hline Tigerella & comprido redondo & redonda & $2-5$ & $\begin{array}{l}5 \mathrm{R} 5 / 10 \mathrm{e} \\
7,5 \mathrm{YR} 6 / 10\end{array}$ & tangerina e vermelha \\
\hline $\mathrm{E}$ & comprido redondo & redonda & 2 & $10 \mathrm{R} 4 / 10$ & tangerina e vermelha \\
\hline K & pêra & redonda & 2 & $5 Y 8 / 12$ & amarela \\
\hline A & cilíndrico & angular & $2-3$ & 5 YR $4 / 8$ & roxa/marrom \\
\hline
\end{tabular}

genética reduzida drasticamente em consequência da domesticação e dos processos de seleção e melhoramento de plantas (Gonçalves et al., 2008). A diversidade genética é considerada mais baixa em cultivares modernas de espécies autógamas, devido a seu sistema de fecundação e também da sua domesticação fora do centro de origem, onde um número limitado de sementes (ou acessos) foi levado pelos exploradores, que hoje servem como a base genética das cultivares modernas (Saavedra \& Spoor, 2002). Além disso, muitos genótipos foram perdidos pela substituição por novas cultivares, ocasionando muitas vezes, o desaparecimento de variedades locais. Em geral, essas novas cultivares apresentam base genética muito estreita, isto é, são muito aparentadas entre si, e a predominância de um restrito número de genótipos ocupando grandes áreas de plantio tem sido considerada um risco para a agricultura, podendo ocasionar a erosão genética (Borém \& Miranda, 2005). O material genético representado pelos acessos estudados contribuirá certamente para aumentar a base genética de futuras cultivares desenvolvidas a partir de cruzamentos que os utilizem.

A existência de coleções de germoplasma com acessos considerados como heirloom precisa ser estimulada e o estudo de caracterização e avaliação desses acessos é valioso para a recomendação aos produtores e para a obtenção de novas cultivares (Rocha et al., 2010).

As baixas similaridades encontradas entre os acessos locais e comerciais, com os heirloom importados, sugerem diversidade genética dentro dos genótipos avaliados, o que foi validado apenas pelos descritores qualitativos. Houve similaridade entre os acessos locais, comerciais e heirloom nas análises com descritores quantitativos.

\section{AGRADECIMENTOS}

À pesquisadora Maria Luiza de Araújo e os funcionários Ivanil Alves dos Santos, Anilton Alves de Souza, Luiz Carlos Gregio, e demais funcionários de campo da Estação Experimental de Seropédica da PESAGRO-Rio, pelo apoio e dedicação prestados no decorrer deste trabalho. À Fundação de Amparo à Pesquisa do Estado do Rio de Janeiro (FAPERJ) pelo apoio financeiro.

\section{REFERÊNCIAS}

ABREU FB; LEAL NR; RODRIGUES R; AMARAL JÚNIOR AT; SILVA DJH. 2004. Divergência genética entre acessos de feijão-de-vagem de hábito de crescimento 
indeterminado. Horticultura Brasileira 22: 547-552.

ALVES RM; GARCIA AAF; CRUZ ED; FIGUEIRA A. 2003. Seleção de descritores botânico-agronômicos para caracterização de germoplasma de cupuaçuzeiro. Pesquisa Agropecuária Brasileira 38: 807-818.

BARROS LM. 1991. Caracterização morfológica e isoenzimática do cajueiro (Anarcadium occidentale), tipos comum e anão precoce, por meio de técnicas multivariadas. Piracicaba: ESALQ. 256p. (Tese doutorado).

BENTO CS; SUDRÉ CP; RODRIGUES R; RIVA EM; PEREIRA MG. 2007. Descritores qualitativos e multicategóricos na estimativa da variabilidade fenotípica entre acessos de pimentas. Scientia Agraria 8: 149-156.

BORÉM A; MIRANDA GV. 2005. Melhoramento de plantas. Viçosa: UFV. 552p.

DAHER RF; MORAES CF; CRUZ CD. 1997. Seleção de caracteres morfológicos em capimelefante (Pennisetum purpureum). Revista Brasileira de Zootecnia 26: 247-259.

DIAS LAS; KAGEYAMA PY; CASTRO GCT. 1997. Divergência genética multivariada na preservação de germoplasma de cacau (Theobroma cacao). Agrotrópica 9: 29-40.

GONÇALVES LSA; RODRIGUES R; SUDRÉ CP; BENTO CS; MOULIN MM; ARAÚJO ML; DAHER RF; PEREIRA TNS; PEREIRA MG. 2008. Divergência genética em tomate estimada por marcadores RAPD em comparação com descritores multicategóricos. Horticultura Brasileira 26: 364-370.

GONÇALVES LSA; RODRIGUES R; AMARAL JÚNIOR AT; KARASAWA M; SUDRÉ CP. 2009. Heirloom tomato gene bank: assessing genetic divergence based on morphological, agronomic and molecular data using a Ward-modified location model. Genetics and Molecular Research 8: 364-374.

IPGRI. 1996. Decriptors for Tomato (Lycopersicon spp.) Rome: Italy. 44p.

JORDAN JA. 2007. The heirloom tomato as cultural object: investigating taste and space. Sociologia Ruralis 47: 20-41.

KARASAWA M; RODRIGUES R; SUDRÉ CP;
SILVAMP; RIVAEM; AMARAL JÚNIORAT. 2005. Aplicação de métodos de agrupamento na quantificação da divergência genética entre acessos de tomateiro. Horticultura Brasileira 23: $1000-1005$.

LEAL MAA; GUERRA JG; PEIXOTO RTG; ALMEIDA DL. 2007. Utilização de compostos orgânicos como substratos na produção de mudas de hortaliças. Horticultura Brasileira 25: 392-395.

MALUF WR; FERREIRA PE. 1983. Análise multivariada da divergência genética em feijão-de-vagem (Phaseolus vulgaris). Horticultura Brasileira 1: 31-34.

NAZAREA VD. 2005. Heirloom Seeds and Their Keepers: Marginality and Memory in the Conservation of Biological Diversity. Tucson: University of Arizona Press. 195p.

OLIVEIRA FJ; FILHO CJA; BASTOS GQ; REIS OV. 2003. Divergência genética entre cultivares de caupi. Pesquisa Agropecuária Brasileira 38: 605-611.

OLIVEIRA MSP; FERREIRA DF; SANTOS JB. 2006. Seleção de descritores para caracterização de germoplasma de açaizeiro para produção de frutos. Pesquisa Agropecuária Brasileira 41: 1133-1140.

OMONHINMIN CA; OSAWARU ME. 2005. Morphological characterization of two species of Abelmoschus: Abelmoschus esculentum and Abelmoschus caillei. Plant Genetic Resources Newsletter 144: 51-55.

PEREIRA AV; VENCOVSKY R; CRUZ CD. 1992. Selection of botanical and agronomical descriptors for the characterization of cassava (Manihot esculenta) germplasm. Revista Brasileira de Genética 15: 115-124.

PEREIRA FHF; PUIATTI M; MIRANDA GV; SILVA DJH; FINGER FL. 2003. Divergência genética entre acessos de taro utilizando caracteres morfoqualitativos de inflorescência. Horticultura Brasileira 21: 520-524.

ROCHA MC; DELIZA R; ARES G; FREITAS DGC; SILVA ALS; CARMO MGF DO; ABBOUD ACS. 2013a. Identifying promising accessions of cherry tomato: a sensory strategy using consumers and chefs. Journal of the Science of Food and Agriculture 93:
1903-1914.

ROCHA MC; DELIZA R; CORREAA FM; CARMO MGF DO; ABBOUD ACS. 2013b. A study to guide breeding of new cultivars of organic cherry tomato following a consumerdriven approach. Food Research International 51: 265-273.

ROCHAMC; GONÇALVES LSA; RODRIGUES R; SILVA PRA; CARMO MGF; ABBOUD ACS. 2010. Uso do algoritmo de Gower na determinação da divergência genética entre acessos de tomateiro do grupo cereja. Acta Scientiarum. Agronomy 32: 423-431.

SAAVEDRA G; SPOOR W. 2002. Genetic base broadening in autogamous crops: Lycopersicon esculentum as a Model. Managing Plant Genetic Diversity 443: 291-299.

SANTOS CAF; OLIVEIRACAV; MENEZES EA. 1995. Seleção de descritores na caracterização e avaliação preliminar de germoplasma de guandu. Pesquisa Agropecuária Brasileira 30: 971-975.

SAS INSTITTUTE INC. 1999. SAS onlinedoc1 version 8. SAS INSTITUTE INC Cary.

STRAPASSON E; VENCOVSKY R; BATISTA LAR. 2000. Seleção de descritores na caracterização de germoplasma de Paspalum sp. por meio de componentes principais. Revista Brasileira de Zootecnia 29: 373-381.

SUDRE CP; LEONARDECZ E; RODRIGUES R; AMARAL JÚNIOR AT; MOURA MCL; GONÇALVES LSA. 2007. Genetic resources of vegetable crops: a survey in the Brazilian germplasm collections pictured through papers published in the journals of the Brazilian Society for Horticultural Science. Horticultura Brasileira 25: 496-503.

SUDRÉ CP; RODRIGUES R; RIVA EM; KARASAWA M; AMARAL JÚNIOR AT. 2005. Divergência genética entre acessos de pimenta e pimentão utilizando técnicas multivariadas. Horticultura Brasileira 23: 22-27.

TRANI PE; PASSOS FA; FOLTRAN DE; TIVELLI SW; RIBEIRO IJA. 2005. Avaliação dos acessos de alho pertencentes à coleção do Instituto Agronômico de Campinas. Horticultura Brasileira 23: 935-939. 